\title{
Endoscopic findings in patients with familial Mediterranean fever and dyspeptic symptoms
}

\author{
Mehmet Agin ${ }^{1}$, Gokhan Tumgor $^{1}$, Aylin Kont ${ }^{2}$, Gulbin Bingol Karakoc ${ }^{2}$, Derya Ufuk Altintas ${ }^{2}$, Mustafa Yilmaz $^{2}$ \\ ${ }^{1}$ Department of Paediatric Gastroenterology, Medical Faculty, Cukurova University Sarıcam, Adana, Turkey \\ 2Department of Paediatric Allergy and Immunology, Medical Faculty, Cukurova University Sarıcam, Adana, Turkey
}

Gastroenterology Rev 2018; 13 (3): 238-241

DOI: https://doi.org/10.5114/pg.2018.76954

Key words: dyspepsia, familial Mediterranean fever, inflammatory bowel disease, MEFV, endoscopy.

Address for correspondence: Mehmet Agin, Department of Paediatric Gastroenterology, Medical Faculty, Cukurova University, 01380 Sarıcam, Adana, Turkey, phone: +90 506801 1083, fax: +90 0322 3387144, e-mail: drmehmet47@yahoo.com

\begin{abstract}
Aim: The aim of the study was to evaluate the clinical, endoscopic, histopathological, laboratory, and imaging findings of patients with familial Mediterranean fever (FMF) presenting with dyspeptic symptoms.

Material and methods: Endoscopy was performed in 28 patients with FMF undergoing colchicine therapy, who presented to our hospital between 2012 and 2014 with abdominal pain, diarrhoea, and dyspeptic complaints. Upper gastrointestinal system endoscopy was performed in all patients; 16 of these underwent colonoscopy. Demographic characteristics, clinical and laboratory data, genetic analysis, imaging data, and endoscopy and colonoscopy findings were evaluated in all patients.

Results: Histopathological analysis of biopsy specimens revealed gastrointestinal pathology in $21(75 \%)$ patients. Upper endoscopy analysis revealed that 14 (50\%) patients had antral gastritis (8 Helicobacter pylori (-) antral gastritis and 6 H. pylori (+) chronic active gastritis) and 2 (7\%) cases had giardiasis. Four antral gastritis cases had concomitant esophagitis, and 2 patients had widespread ulcers in the terminal ileum. The rest had a normal colonic appearance; however, three patients with normal colonoscopic findings were found to have chronic active colitis on histopathological examination.

Conclusions: In this study, most patients with FMF presenting with dyspeptic complaints had gastrointestinal pathology. Furthermore, there was higher inflammatory bowel disease incidence in these patients.
\end{abstract}

\section{Introduction}

Familial Mediterranean fever (FMF) is an autosomal recessive genetic disease characterised by recurrent febrile episodes and serous membrane inflammation [1]. MEFV, the FMF gene, is located on the short arm of chromosome 16 [2]. Familial Mediterranean fever is frequently observed in individuals of Turkish, Jewish, or Asian descent, and its diagnostic criteria are well-defined in the literature [3]. Although abdominal pain is the most frequently observed FMF symptom, patients may present with other symptoms, such as fever and joint pain [4].

Abdominal pain may be observed because of FMF-related vasculitis, inflammatory bowel disease (IBD), or irritable bowel syndrome or may occur as a side-effect of colchicine therapy. Other gastrointestinal system (GIS) symptoms in addition to acute abdominal attacks have been described in patients with
FMF. For example, in addition to an acute attack or its indicators such as acute peritonitis and intestinal obstruction, patients may experience symptoms unrelated to an FMF attack, which may include functional bowel disorders such as constipation and diarrhoea. These patients may experience symptoms associated with amyloidosis; ischaemic colitis and perforation can result from amyloid deposition in the colon [4]. Although some studies have reported that patients with FMF had a greater incidence of functional abdominal pain, functional dyspepsia, and irritable bowel syndrome than healthy individuals, this has not yet been conclusively demonstrated.

Inflammatory bowel disease is characterised by irregular GIS mucosal immune response. The pathogenesis underlying IBD has not been completely elucidated; however, environmental, genetic, and immune factors; endogenous and exogenous triggers; and complex inter- 
actions within the intestinal flora have been shown to play important roles [5]. Crohn's disease (CD) and FMF share common clinical and pathological features such as arthritis, abdominal inflammation, recurrence, and abnormal apoptotic regulation. Although the role of MEFV mutation as a major modifier in CD is controversial, various groups reported that CD was more prevalent in patients with FMF than in the general population [6-9]. Familial Mediterranean fever attacks and amyloidosis-related complications were reported more frequently in patients with FMF with concomitant CD [7].

\section{Aim}

Although numerous studies suggested a potential correlation between FMF and IBD, to date, limited direct evidence is available. In this study, we assessed intestinal inflammation using clinical, endoscopic, histopathological, laboratory, and imaging studies in patients with FMF presenting with dyspeptic complaints; this enabled us to evaluate several previously undocumented features of FMF.

\section{Material and methods}

Twenty-eight patients diagnosed with FMF according to the Tel Hashomer criteria, who presented to the Paediatric Gastroenterology Clinic at Cukurova University Faculty of Medicine, Turkey, with dyspeptic complaints between 2012 and 2014 were enrolled in this study. The protocol was approved by the Ethics Committee of Cukurova University. The demographic and clinical features, family history, nutritional status, comorbidities, and presence of perianal lesions were recorded for all patients. All patients reported symptoms of abdominal pain, diarrhoea, dyspepsia, bloating, fever, nausea, vomiting, burping, weight loss, and joint pain despite colchicine therapy. All serologic tests were performed at the Cukurova University Hospital core laboratory using standard methods and included complete blood count, biochemical panel, C-reactive protein, erythrocyte sedimentation rate, fibrinogen, antineutrophilic cytoplasmic antibody, and anti-saccharomyces cerevisiae antibody. All patients underwent ultrasonography (USG), computed tomography (CT), and intestinal and colonic X-ray series with barium and were evaluated for ocular lesions by ophthalmological examination. MEFV mutations and HLA-B5 status of all patients enrolled in the study were assessed by polymerase chain reaction sequence-specific oligonucleotide method (Luminex ${ }^{\circledast}$, Austin, TX, USA). After routine bowel cleansing preparations, the colonoscopies were performed with a Pentax EG-2730 K gastroscope and a colonoscope (Pentax, Tokyo, Japan). Twenty-eight cases underwent endoscopy (12 gastroscope and 16 gastroscope and colonoscopy) using a gastroscope and a colonoscope. For sedation, standard doses of propofol and midazolam were used. Viral, bacterial, and parasitic infections; Behcet's disease; tuberculosis; immune deficiencies; and food allergy were ruled out by clinical and laboratory findings in patients with inflammation identified using colonoscopy and histopathology. The cases enrolled were evaluated for their demographic and clinical features, laboratory and genetic analysis, imaging methods, and endoscopy and colonoscopy procedures.

\section{Results}

Nineteen (68\%) patients were female and 9 (32\%) were male. All patients indicated abdominal pain; nausea, vomiting, burping, diarrhoea, and weight loss were reported in 18 (78\%), 10 (43\%), 3 (13\%), 4 (17\%), and $4(17 \%)$ patients, respectively (Table I). None of the patients had perianal lesions. Stool cultures were negative in all four patients with diarrhoea; furthermore, no pathology was observed by stool microscopy. Abdominal USG identified biliary sludge in one case and nephrolithiasis in another patient. None of the patients had proteinuria or histopathological amyloidosis. Ophthalmological examination was normal in all cases. M694V mutation within MEFV was found in $6(22 \%)$ patients, whereas 4 out of 5 (80\%) patients diagnosed with IBD were homozygous for the M694V mutation. Only one patient with FMF and IBD had a relative with FMF.

Histopathological examination of the samples obtained by endoscopy and/or colonoscopy identified pathology in 21 (75\%) patients. Specifically, 14 (50\%) patients had antral gastritis; $H$. pylori (-) chronic gastritis and $H$. pylori $(+)$ chronic active gastritis were found in

Table I. Patient complaints and demographic findings $(N=28)$

\begin{tabular}{lc}
\hline Complaints & Results \\
\hline Abdominal pain & $28(100 \%)$ \\
\hline Nausea & $18(78 \%)$ \\
\hline Vomiting & $10(43 \%)$ \\
\hline Joint pain & $6(22 \%)$ \\
\hline IBD diagnosis & $5(18 \%)$ \\
\hline Diarrhoea & $4(17 \%)$ \\
\hline Weight loss & $4(17 \%)$ \\
\hline Burping & $3(13 \%)$ \\
\hline Family history & $1(4 \%)$ \\
\hline Rectal bleeding & 0 \\
\hline Bloody diarrhoea & 0 \\
\hline Perianal disease & 0
\end{tabular}

IBD - Inflammatory bowel disease. 
Table II. Upper GIS endoscopy and colonoscopy findings

\begin{tabular}{cccc}
\multicolumn{2}{c}{ Upper GIS endoscopy appearance $(n=28)$} & \multicolumn{2}{c}{ Colonoscopy appearance $(n=16)$} \\
\hline Antral gastritis & $10(36 \%)$ & Ulcer in terminal ileum & $2(12 \%)$ \\
\hline Esophagitis & $2(7 \%)$ & Normal & $14(88 \%)$ \\
\hline Antral gastritis and esophagitis & $4(14 \%)$ & & \\
\hline Normal & $12(43 \%)$ & &
\end{tabular}

8 and 6 patients, respectively. In addition, 4 cases with antral gastritis had concomitant esophagitis, and 2 (7\%) cases were positive for giardiasis. Of these, histopathological examination was normal in 12 cases. Although the appearance of colon was normal in all cases, two patients had widespread ulcers in the ileum (Table II). Five out of a total of 16 (31\%) patients who underwent colonoscopy had findings indicative of IBD by histopathological examination of samples obtained from the colon and terminal ileum; goblet cell loss, cryptitis, and cryptic hyperplasia in these specimens indicated active colitis suggestive of IBD, whereas colonoscopy and histopathology in the remaining 11 (69\%) patients were normal (Figures 1 and 2).

\section{Discussion}

Although FMF diagnosis is achieved by clinical evaluation, patients with FMF with comorbidities such as IBD and vasculitis may present with different symptoms [4]; in addition to abdominal pain, other GIS-related symptoms can be observed in patients with FMF. Furthermore, although these symptoms may occur as complications of FMF and amyloidosis, they may arise from comorbidities $[10,11]$. There are only a limited number of studies investigating endoscopic findings in patients with FMF. Gurkan and Dalgic et al. reported 11 cases with FMF, of which two had pangastritis and one had prepyloric ulcer [12]. In another study, Ozaltun et al. investigated the correlation between $H$. pylori and FMF attacks and reported that out of 85 patients diagnosed with FMF, 31 had $H$. pylori

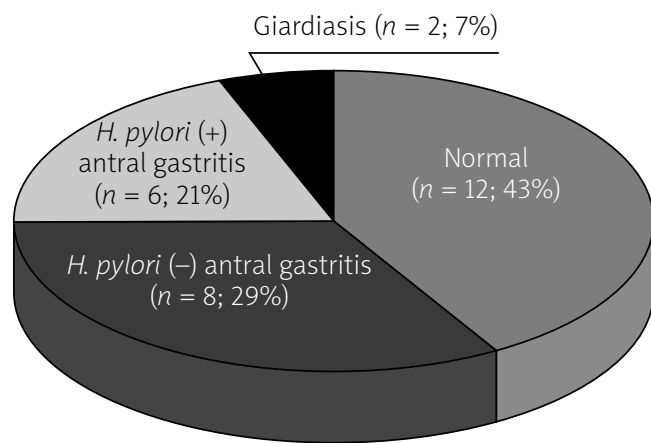

Figure 1. Histopathology finding of patients who underwent upper gastrointestinal system endoscopy
IgG seropositivity by urea respiration test and that endoscopic and histopathological examination revealed pathology in 13 of these cases [13]. Tuzun et al. determined H. pylori positivity in $30(67.6 \%)$ patients in a cohort of 44 FMF cases; the prevalence of $H$. pylori infection in these patients did not differ from that in the normal population [14]. The histopathological $H$. pylori positivity level in our study was $24 \%$, which was not particularly high and was similar to that found in children in Turkey $[15,16]$.

Studies previously demonstrated a close association between IBD and FMF. In one study, CD was diagnosed in only seven out of 4978 patients with FMF, with a prevalence of 5.6/100,000 [9]. The Turkish FMF study group reported IBD in only $4(0.1 \%)$ out of 2716 cases with FMF [17]. Inflammatory bowel disease and FMF both present with recurrent and periodic symptoms, and both MEFV and NOD2/CARD15, the predicted CD-associated gene, are located on chromosome 16. Furthermore, protein products of these genes, pyrin and NOD2/CARD15, are structurally similar and belong to the same apoptosis-regulating protein family, with key roles in apoptosis regulation, cytokine processing, and inflammation. Recent studies have highlighted the role of pyrin as an important component of the inflammasome, which is a critical regulator of inflammation that is responsible for the production of IL-1 [18]. MEFV mutations trigger inflammation and result in increased transcription of proinflammatory cytokines such as tumour necrosis factor- $\alpha$, IL-6, and IL-8 [18], whereas NOD2/CARD15 functions during innate immunity [19]. Thus, dysregulations of one or both of these proteins with closely associated pathways may affect the clin-

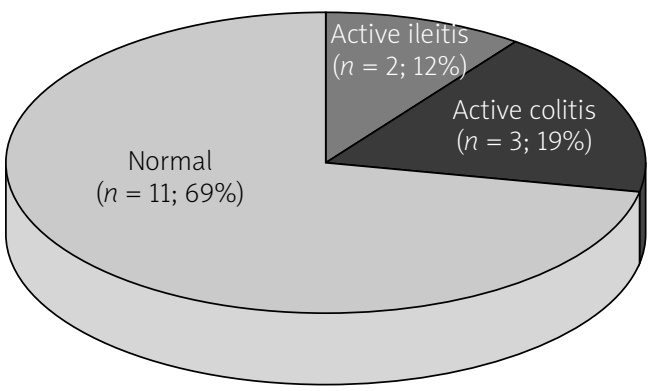

Figure 2. Histopathology finding of patients who underwent colonoscopy 
ical manifestation of either disease and may partially explain the significantly increased frequency of MEFV mutations in CD patients compared with those with ulcerative colitis. Thus, further elaboration of the role of pyrin in inflammation control in healthy and diseased individuals will advance our understanding of the role of MEFV mutations in IBD patients. In our study, 14 out of 16 cases undergoing colonoscopy had normal appearance of the colon, and multiple, widespread ulcers were observed in the ileum in only 2 cases. In addition, 3 patients with normal colonoscopic appearance were found to have chronic active colitis by histopathological examination. Although chronic active colitis observed in three patients may be associated with IBD, FMF-related bowel inflammation should not be excluded because of the normal appearance of the mucosa. The symptoms that were resistant to colchicine in these patients improved rapidly with mesalazine therapy. These patients were diagnosed as CD and were started on steroid therapy with dramatic improvements. Finally, in our study, M694V mutations were higher in FMF patients with IBD (80\%).

\section{Conclusions}

Familial Mediterranean fever is a very common disease in Turkey, and GIS-related pathology is present in cases presenting with dyspeptic complaints. The prevalence of IBD in FMF cases has increased. Biopsy of FMF cases with normal colonoscopic findings is important to determine histopathological inflammation that may not be evident by gross examination, as evidenced by increased lymphocytic and polymorphonuclear cell infiltration in the lamina propria in 5 patients with FMF with IBD in our cohort. The presence of goblet cell loss, crypt hyperplasia, and active chronic infection primarily located in the submucosa were highly suggestive of IBD and were observed in most of our patients; thus, IBD should be considered as comorbidity in patients with FMF with atypical clinical findings resistant to treatment.

\section{Conflict of interest}

The authors declare no conflict of interest.

\section{References}

1. John CC, Gilsdorf JR. Recurent fever syndrome in children. Ped infect Dis J 2002; 21: 1071-7.

2. Frenkel J, Kuis W. Overt and occult rheumatic diseases: the child with chronic fever. Best Pract Res Clin Rheumatol 2002; 16: 443-69.

3. Mor A, Gal R, Livneh A. Abdominal and digestive system associations of familial Mediterranean fever. Am J Gastroenterol 2003; 98: 2594-604.

4. Drenth JPH, van der Weer JW. Hereditary periodic fever. N Engl J Med 2001; 345: 1748-57.
5. Dubinsky M. Special issues in pediatric inflammatory bowel disease. World I Gastroenterol 2008; 14: 413-20.

6. Cattan D, Notarnicola C, Molinari N, Touitou I. Inflammatory bowel disease in non-Ashkenazi Jews with familial Mediterranean fever. Lancet 2000; 355: 378-9.

7. Fidder H, Chowers Y, Lidar M, et al. Crohn's disease in patients with familial Mediterranean fever. Medicine 2002; 81: 411-6.

8. Fidder H, Chowers Y, Ackerman Z, et al. The familial Mediterranean fever (MEVF) gene as a modifier of Crohn's disease. Am J Gastroenterol 2005; 100: 338-43.

9. Giaglis S, Mimidis K, Papadopoulos V, et al. Increased frequency of mutations in gene responsible for familial Mediterranean fever (MEFV) in a cohort of patient with ulcerative colitis: evidence for a potential disease-modifying effect? Dig Dis Sci 2006; 51: 687-92.

10. Brik R, Litmanovitz D, Berkowitz D, et al. Incidence of familial Mediterranean fever (FMF) mutations among children of Mediterranean extraction with functional abdominal pain. J Pediatr 2001; 138: 759-62.

11. Börekci E, Celikbilek M, Soyturk M, et al. Functional gastrointestinal disorders in patient with familial Mediterranean fever. Int J Rheum Dis 2017; 20: 2101-5.

12. Gurkan OE, Dalgic B. Gastrointestinal mucosal involvement without amyloidosis in children with familial Mediterranean fever. J Pediatr Gastroenterol Nutr 2013; 57: 319-23.

13. Ozaltun F, Bakkaloğlu A, Saltik IN, et al. Helicobacter pylori infection in the Turkish children with familial Mediterranean fever: is it a cause of persistent inflamation? Clin Rheumatol 2004; 23: 186-7.

14. Tuzun A, Erdil A, Yeşilova Z, et al. Helicobacter pylori infection in patients with familial Mediterranean fever. Turk Klin J Gastroenterohepatol 2003; 14: 82-5.

15. Selimoglu MA, Ertekin V, Inandi T. Seroepidemiology of Helicobacter pylori infection in children living in eastern Turkey. Pediatr Int 2002; 44: 666-9.

16. Ozen A, Ertem D, Pehlivanoglu E. Natural history and symptomatology of Helicobacter pylori in childhood and factors determining the epidemiology of infection. J Pediatr Gastroenterol Nutr 2006; 42: 398-404.

17. Tunca M, Akar S, Onen F, et al.; Turkish FMF Study Group. Familial Mediterranean fever (FMF) in Turkey: results of a nationwide multicenter study. Medicine 2005; 84: 1-11.

18. Chae JJ, Wood G, Masters SL, et al. The B30.2 domain of pyrin, the familial Mediterranean fever protein, interacts directly with caspase-1 modulate IL-1 beta production. Proc Natl Acad Sci USA 2006; 103: 9982-7.

19. Church LD, Cook GP, McDermott MF. Primer: inflammasomes and interleukin 1 beta in inflammatory disorders. Nat Clin Pract Rheumatol 2008; 4: 34-42.

Received: 23.02.2018

Accepted: 12.06 .2018 